\title{
Kinetics of Tethered Ligands Binding to a Surface Receptor
}

\author{
Samuel Bell and Eugene M. Terentjev*(1) \\ Cavendish Laboratory, University of Cambridge, Cambridge CB3 OHE, U.K.
}

\section{Supporting Information}

ABSTRACT: The rate of binding of a grafted polymer to the surface is controlled by entropic barriers. Using a mean-field approximation of ideal polymer dynamics, we first calculate the characteristic binding time for a tethered ligand reaching for a binding site located on the tethering surface. This time is determined by two separate entropic effects: a barrier for the chain to be stretched sufficiently to reach the distant target and a restriction on chain conformations near the surface, versus the increase in available phase space for longer chains. The competition between these two constraints determines the optimal (shortest) binding time. The theory is then extended to model bridging between two surfaces, in particular relevant for cell adhesion. Here the tethered ligand reaches for a receptor on a parallel surface, and the binding time depends

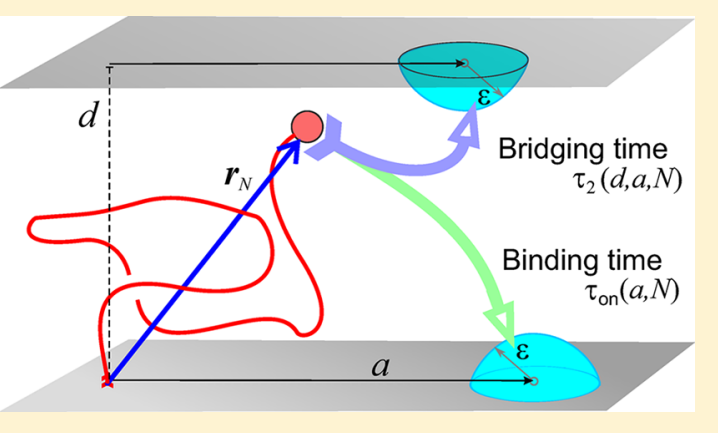
on the gap between the two constraining surfaces. Again, an optimal binding time is determined for the given tether geometry. The results look similar to those for free particles in the "narrow escape problem", but modified by an entropic activation factor introduced by the tether.

\section{INTRODUCTION}

Ordered self-assembly requires the ability to organize and bind many molecules into a coherent structure. In biology, most selfassembling structures rely on specific interactions, matching ligands, and distinct binding sites. The kinetics of self-assembly is a broad and rich topic, which offers a fundamental understanding of processes being used in the construction of structured and functional aggregates.

One such process is the binding of a tethered ligand to a binding site. This is a fundamental process, involved in many biological settings. The thrombin receptor, a transmembrane protein, activates by cleaving an amino-terminal extension on the extracellular side of the membrane, unmasking a ligand which binds to another part of the receptor nested against the cell membrane. ${ }^{1-3}$ Cytoskeletal molecular motors (dynein, kinesin, and myosin) all require a periodic binding of their ligand at the end of a flexible "arm" to a site on a substrate (microtubule or F-actin, respectively) a certain distance away., ${ }^{4,5}$ There is also a larger scale process of cell-cell or cell-surface adhesion. Jeppesen et al. ${ }^{6,7}$ examined this problem for one specific binding site, where ligands tethered to the cell surface by flexible chains could also associate with the matching receptor on an adjacent cell. They found a dependence on the configuration of the polymer tether: in particular, how often the chains entered extended configurations to reach the distant receptors. Their treatment did not extend to an analytical expression of the binding rate. Theoretically solving this problem is one of our main tasks here.

The search for a small target has already been considered in the context of DNA looping, ${ }^{8-10}$ where the mean first time for two distant monomers on a polymer chain to meet was calculated. Such loops are observed experimentally in chromatin $^{11,12}$ and in surface-tethered DNA. ${ }^{13,14}$ In fact, our calculation is based on the ideas of Szabo et al., ${ }^{9}$ although in their problem of forming a loop the distance to the binding site is zero, and accordingly no activation exponential has been observed. Our problem is also similar to the "narrow escape problem". ${ }^{15-18}$ Here a Brownian particle is confined to a domain whose boundary is entirely reflecting, apart from a small absorbing patch. The "narrow escape time" is the mean first time the particle reaches the absorbing patch and escapes the volume it was diffusing in. The diffusion of a tethered particle (ligand) is different, since it is confined by the polymer chain statistics rather than hard boundaries.

In our model, this graft to the surface is persistent; we regard the polymer tether as fixed at the origin. The remaining free chain has a second binding site, subject to thermal motion. The chain has a hard constraint of the wall to which it is grafted to, but its free end (with the binding ligand) also has a soft constraint on how far it can extend from the grafted origin. If the chain end-to-end distance increases, there will be a resulting reduction in its entropy, which leads to an entropic barrier for associated activated processes. Such entropic barriers have been investigated in polymer dynamics ${ }^{19}$ and in colloid glassy dynamics. ${ }^{20}$ They have an important role to play in cell and molecular biology: entropic barriers show up in polymer translocation through a pore ${ }^{21,22}$ as well as the looping time of a polymer chain. ${ }^{9}$ They also play a role in the protein aggregation into amyloids ${ }^{23}$ and in more general protein folding funnel problems. $^{24}$

Received: August 14, 2017

Revised: October 4, 2017

Published: October 18, 2017 
In this paper, we examine a trade-off in the entropic barrier faced by reaching the distant target, which is on the same surface a fixed distance $a$ away, against the reduction in chain confinement. We calculate the mean first time it takes the chain to find the receptor, which is determined by an activation law where the effective potential barrier is purely entropic, $-T \Delta S$. As such, the explicit temperature disappears from the Boltzmann factor, and the mean binding time is proportional to $\exp \left[a^{2} / R_{\mathrm{g}}{ }^{2}\right]$, with $R_{\mathrm{g}}$ the radius of gyration of the tethered chain. If we make the chain very short, there will be a very small chance of it stretching far enough to reach the receptor site, and the time to reach the receptor will be long. If we make the chain very long, there will no longer be such a high entropic penalty for reaching the same receptor; however, the chain will now be able to explore a very large volume, and that reduces the probability that the binding site will hit the target. Once the expression for the average binding time is obtained, we are able to find the optimal chain length for the fastest binding time: this turns out to be exactly when the target separation $a$ is equal to the radius of gyration of the chain. The second aspect of this problem is addressed in the last section of the paper: we examine binding of the tethered ligand to a receptor on a different surface, across a gap $d$, in a geometry directly related to cell-cell adhesion of Jeppesen. ${ }^{6}$ Again, we find the entropic barrier defining the activation rate of such a process and how the rate of such bridging across the gap depends on the distance d.

\section{DIFFUSION OF A TETHERED LIGAND}

We consider an ideal polymer chain: $N$ segments of length $b$, grafted at the origin to a flat surface, where the last $(\mathrm{Nth})$ monomer is the binding ligand. To find the equilibrium distribution of the chain configuration, we use the Gaussian chain propagator of an ideal chain $G_{N}\left(\mathbf{r}, \mathbf{r}_{0}\right)$ : the probability that such a chain begins at $\mathbf{r}_{0}$ and ends at $\mathbf{r}^{25}$ We need to implement a boundary condition on the substrate plane $z=0$. This is a question with a very long history, ${ }^{26}$ culminating with the classical work of Edwards and Freed ${ }^{27}$ on the properties of confined chains. Many aspects of this problem, of a chain near a hard wall, were explored over the years, with seminal contributions $^{28-30}$ being just a few of many important references, all using and exploiting the "exclusion" condition: $\left.G_{N}\left(\mathbf{r}, \mathbf{r}_{0}\right)\right|_{\text {surface }}=0$. This means that no monomer may rest against the wall. Surprisingly, this restriction is not well covered in the literature, and it is difficult to acquire intuition for it. Exclusion seems drastically different from the reflecting boundary condition one would impose on Brownian particles, if they were not connected on the chain. This is a subtle, yet potent effect of chain configurational entropy-understood first by DiMarzio from the point of view of counting restricted chain configurations $^{26}$ and then by Edwards and Freed by looking at the entropic repulsive force arising if we were to push the chain into a wall. ${ }^{27}$

When only one planar wall is present, the Gaussian chain propagator can satisfy the boundary condition by adding one negative chain "image". Although we tether the chain at the origin $\mathbf{r}=0$, it is necessary to insist that the first monomer steps directly away from the surface, so $z_{0}=b$, and the image chain starts at $\bar{z}_{0}=-b$. The remaining chain is then of length $N-1$, but since we must assume $N$ is large, we ignore this:

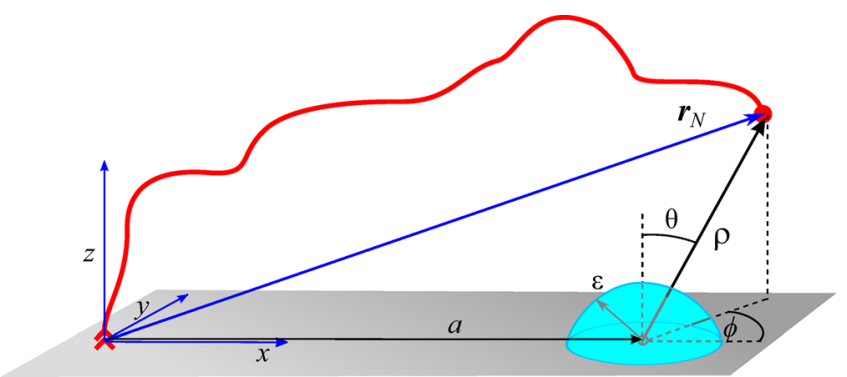

Figure 1. A chain of $N$ monomers is tethered to a hard wall at the origin. A hemispherical absorbing target of radius $\varepsilon$ lies on the same surface, a distance $a$ from the tether. We look for the equilibrium radial probability $P_{\mathrm{eq}}(\rho)$ for the ligand distance to the target.

$$
G_{N}(\mathbf{r})=\left(\frac{3}{2 \pi N b^{2}}\right)^{3 / 2}\left[\mathrm{e}^{-3(\mathbf{r}-b \hat{\mathbf{z}})^{2} / 2 N b^{2}}-\mathrm{e}^{-3(\mathbf{r}+b \hat{\mathbf{z}})^{2} / 2 N b^{2}}\right]
$$

The binding ligand (located at $\mathbf{r}_{N}$ ) needs to find a surface receptor placed a distance $a$ from the grafting site, as illustrated in Figure 1. The receptor zone is assumed hemispherical, with a small radius $\varepsilon$. We will now construct an effective radial probability distribution $P_{\mathrm{eq}}(\rho)$ for the distance $\rho$ from the binding site $\mathbf{r}_{N}$ to the target receptor.

In eq 1 , the propagator for the position of the chain end is presented using a Cartesian coordinate system with the origin at the point of grafting. However, since we are looking for the passage time into a hemisphere centered on the receptor, it is useful to switch to spherical polar coordinates $(\rho, \theta, \phi)$ centered on the target (Figure 1). Then we will need to integrate over the two angles to finally derive the radial probability density about the target receptor, $P_{\text {eq }}(\rho)$, which will be a function of the receptor position $a$. Let us choose the target to be in the positive $x$-direction relative to the tethered end. Then the coordinate transformation is

$$
x-a=\rho \sin \theta \cos \phi, \quad y=\rho \sin \theta \sin \phi, \quad z=\rho \cos \theta
$$

The two scalar products in the combined exponents of eq 1 become

$$
(\mathbf{r} \pm b \hat{\mathbf{z}})^{2}=a^{2}+b^{2}+\rho^{2}+2 a \rho \sin \theta \cos \phi \pm 2 b \rho \cos \theta
$$

The next step of integration over the solid angle on the unit hemisphere is not easy. We need to evaluate

$$
I=\int_{0}^{\pi / 2} \mathrm{~d} \theta \sin \theta \int_{0}^{2 \pi} \mathrm{d} \phi \mathrm{e}^{-\alpha \cos \phi \sin \theta \pm \beta \cos \theta}
$$

where parameters $\alpha$ and $\beta$ involve $N, b, a$, and $\rho$. This is solved by realizing that the integrand has a nontrivial axial symmetry. Exploiting this symmetry, we transform back into Cartesian coordinates about the target- $x^{\prime}=\cos \phi \sin \theta, z^{\prime}=\cos \theta$-and rotate these new coordinates by an angle $\varphi=\tan ^{-1}(\beta / \alpha)=$ $\tan ^{-1}(b / a)$ around the $y$-axis. The direction of this rotation depends on the sign of the $z$-term in the exponent (i.e., whether we are dealing with the "real" or "image" Gaussian term). The details of this calculation are given in Supporting Information part $\mathrm{A}$, including the full expression for the normalized radial distribution function $P_{\mathrm{eq}}(\rho)$. It turns out that a very good approximation exists to that complicated expression, which is nearly accurate except for the region $a \leq b$ (which we are not interested in here): 


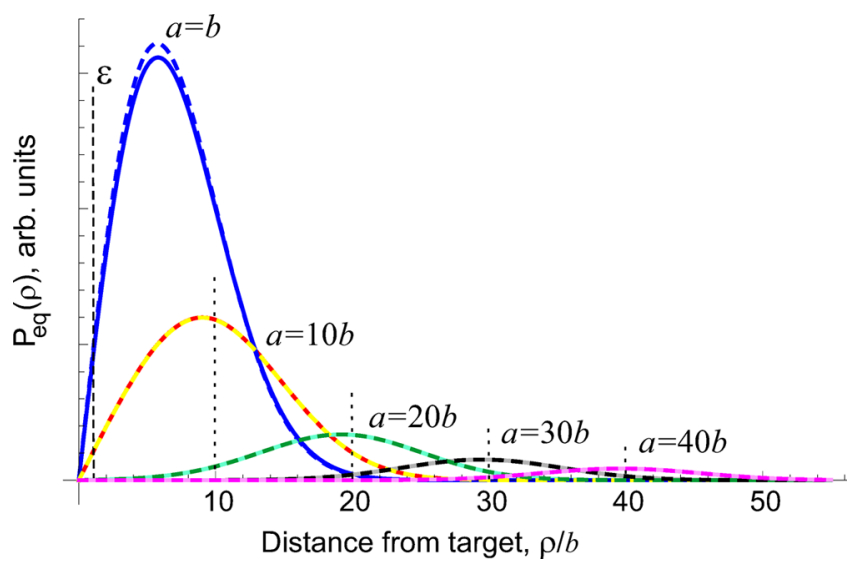

Figure 2. Radial probability density $P_{\mathrm{eq}}(\rho)$, given by eq 5 , for $N=100$ (so $R_{\mathrm{g}}=10 b$ ) and for several values of the target position: $a / b=1,10$, 20, 30, and 40. Dashed lines show the exact result of the angular integration (eq 4) for comparison: the deviations are only seen at small $a$.

$$
P_{\mathrm{eq}}(\rho) \approx \frac{3}{N b^{2} a} \mathrm{e}^{-3\left(a^{2}+\rho^{2}\right) / 2 N b^{2}} I_{1}\left(\frac{3 a \rho}{N b^{2}}\right)
$$

where $I_{1}(\ldots)$ is the first rank modified Bessel function of the first kind. Note that the thermodynamic partition function of a grafted Gaussian chain is $\sqrt{6 / N \pi}$ in our notations. It is useful to define a length-scale parameter $R_{\mathrm{g}}=\sqrt{ } \mathrm{Nb}$, closely related to the chain radius of gyration. ${ }^{25}$

This distribution is plotted in Figure 2. The probability density goes to zero as $\rho \rightarrow 0$ because of exclusion at the surface and then peaks before decaying away again due to the chain being overstretched. The peak of this distribution is going to be close to the target distance, $\rho \approx a$, because the chain is most likely to be found near the tether (which is a distance $\rho=$ $a$ away from the target). The actual peak lies at just less than $\rho$ $=a$, as a result of averaging over the polar angles, but the difference becomes less significant as $a \gg R_{\mathrm{g}}$.

One can identify the radial probability density discussed above with an effective radial potential via the Boltzmann factor: $V_{\text {eff }}=-k_{\mathrm{B}} T \ln \left[\rho^{2} P_{\text {eq }}(\rho)\right]$. The resulting effective potential that the binding ligand on the $N$ th chain segment experiences is a function of distance from the target receptor and depends on two relevant length scales in the problem: the chain radius of gyration $R_{\mathrm{g}}=N^{1 / 2} b$ and the distance to target $a$. It is plotted in Figure 3 for several values of $a$ : above and below the $R_{\mathrm{g}}$. The effective potential has a minimum (seen as the peak of the radial probability distribution) but diverges in the close proximity to the target because of the exclusion boundary condition the wall imposes on the chain: this produces an effective (entropic) repulsion that the ligand has to overcome to reach the target at $\rho \rightarrow 0$. We see in Figure 3 that this effective energy barrier between the minimum of $V_{\text {eff }}$ and the value at $\rho=\varepsilon$ is about $4 k_{\mathrm{B}} T$ for $a=b$, rising to almost $20 k_{\mathrm{B}} T$ for $a=30 b$, for the chain of 100 monomers.

\section{MEAN FIRST PASSAGE TIME TO TARGET}

To find the reaction rate, we could convert our probability distribution into an effective potential, and then use Kramers theory $^{31,32}$ to derive an expression for the rate.

However, since we already have the equilibrium probability distribution for the single radial variable $\rho$ (the distance of the dangling ligand from the target receptor), we can instead use a

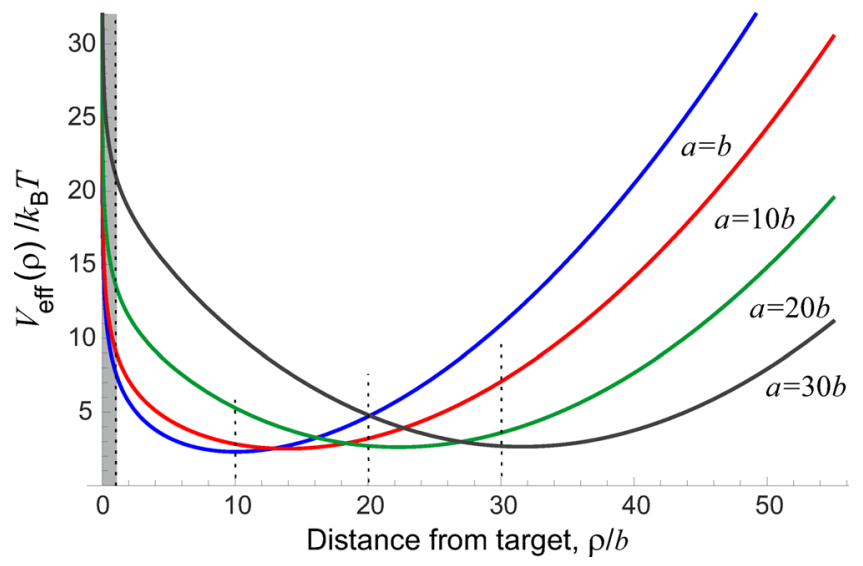

Figure 3. Effective potential $V_{\text {eff }}(\rho)$ of the end of the chain, plotted for $N=100$ (so $R_{\mathrm{g}}=10 b$ here), and several values of the target position: $a / b=1,10,20$, and 30 . The shaded area around the origin marks the receptor size $\varepsilon=b$.

famous relation derived by Szabo et al. ${ }^{9}$ to find the mean first passage time (MFPT) to an absorbing surface at $\rho=\varepsilon$ :

$$
\tau_{\mathrm{abs}}=\int_{\varepsilon}^{\infty} \frac{\mathrm{d} \rho}{D \rho^{2} P_{\mathrm{eq}}(\rho)}\left[\int_{\rho}^{\infty} \mathrm{d} \rho^{\prime} \rho^{\prime 2} P_{\mathrm{eq}}\left(\rho^{\prime}\right)\right]^{2}
$$

where we assume the diffusion coefficient of the free end of the chain, $D=k_{\mathrm{B}} T / \gamma$, is constant and equal to the diffusion coefficient of a single monomer in solution (in the spirit of the Rouse $\operatorname{model}^{25}$ ). In the free particle case, it is necessary to constrain the particle with an upper reflective boundary, but for the polymer chain, the entropic spring effect ensures the integrals converge if we take the upper limit to $\rho=\infty$.

Even for the approximate probability distribution given in eq 5 , the integral in eq 6 does not have an easy analytical solution. However, it is clear from eq 6 that the bracketed integral will have a value between 0 and 1 (with the integral equal to 1 for $\rho$ $=0$, since $P_{\text {eq }}$ is normalized). We also notice the probability appearing in the denominator of the final integral. This means that the mean first-passage time will be dominated by any regions where $\rho^{2} P_{\mathrm{eq}}(\rho) \rightarrow 0$. For the tethered chain, this does occur as $\rho \rightarrow 0$ (as is obvious from Figure 2), and so the main contribution to the mean first passage time comes from the region of small $\rho$ (where the bracketed integral can be approximated as 1). Expanding the integrand about $\rho=0$ and retaining only the leading term, we find that eq 6 reduces to a simple integral

$$
\tau_{\text {on }} \approx \frac{2 N^{2} b^{4}}{9 D} \mathrm{e}^{3 a^{2} / 2 N b^{2}} \int_{\varepsilon}^{\infty} \frac{\mathrm{d} \rho}{\rho^{3}}=\frac{N^{2} b^{4}}{9 D \varepsilon^{2}} \mathrm{e}^{3 a^{2} / 2 N b^{2}}
$$

where, as before, we recognize the characteristic length scale $R_{\mathrm{g}}$ $=N^{1 / 2} b$ : the radius of gyration of an ideal chain. Equation 7 is the first of two main results of this paper. We denote this characteristic time of binding to a receptor on the same surface as $\tau_{\text {on }}$.

The comparison between the exact numerical integral and the approximation presented in eq 7 is shown in Figure 4, where the mean time of the binding ligand reaching the target receptor is plotted against the "size" of the receptor (measured by the radius of the hemisphere $\varepsilon$; see the sketch in Figure 1). The deviations are enhanced in the Figure 4 inset by the logarithmic scale and are evidently very small for sufficiently 

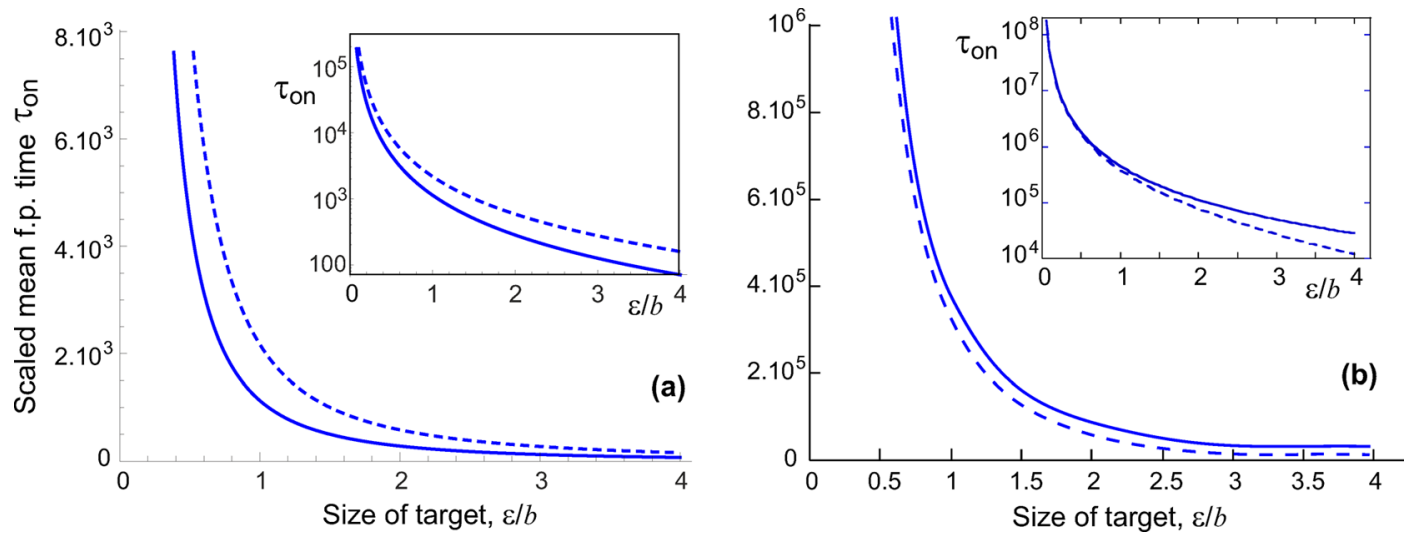

Figure 4. Mean first passage time $\tau_{\text {on }}$ (in units of $b^{2} / D$ ) calculated numerically from eq 6 (dashed lines), compared with the approximation of eq 7 (solid lines). Here, again, $N=100$ (so $R_{\mathrm{g}}=10 b$ ); plot (a) is for the close proximity of the receptor, $a=b$, and plot (b) a distant receptor, $a=20 b$. In reality, one might imagine the relevant size of targets for specific binding to be not much greater than $b$. The insets show the same plots on logarithmic scale, which covers a greater range and also emphasizes the deviations of the approximate expression for $\tau_{\mathrm{on}}$.

small targets. Clearly, eq 7 is a good approximation, offering a compact analytical expression that we can examine.

\section{BRIDGING ACROSS A GAP}

During cell-cell adhesion, the two cell membranes come very close to one another. Thus, they can be modeled as two parallel planes a distance $d$ apart, with their actual curvature playing a minor role in the dynamics. As in the single plane case of the previous sections, we must consider the first monomer as stepping directly away from the surface, so the tether in this coordinate system is at $(0,0, b)$. We can then write down the chain propagator in exactly the manner of Edwards and Freed, ${ }^{27}$ separating the unconstrained chain in the $x y$-plane from the narrow confining box along $z$, with one chain end fixed at $z=b$ :

$$
\begin{aligned}
& G_{N}(x, y, z)=\frac{2}{d} \sum_{n=1}^{\infty} \sin \left(\frac{n \pi z}{d}\right) \sin \left(\frac{n \pi b}{d}\right) \mathrm{e}^{-n^{2} \pi^{2} N b^{2} / 6 d^{2}} \\
& \times \frac{3}{2 \pi N b^{2}} \mathrm{e}^{-3\left(x^{2}+y^{2}\right) / 2 N b^{2}}
\end{aligned}
$$

As before, both planes are monomer excluding due to the chain entropic repulsion. When cells are close to one another, the distance $d^{2} \ll N b^{2}$, we are free to consider only the first term in the sum, as the exponential in the sum suppresses subsequent terms (a regime known as the ground-state dominance in polymer physics).

As in the single-plane case, the key is to derive the radial probability distribution $P_{\text {eq }}(\rho)$ for the ligand a distance $\rho$ away from the binding site (see Figure 5). If the target receptor is placed a perpendicular distance $a$ from the tether, on the opposite plane, then there is no obvious symmetry to exploit. Instead, it is possible to make progress if we use the approximation $a \gg \varepsilon$. The propagator is radial in the $x y$ plane about the tether. If the receptor is placed in the $x$ direction, then around the receptor, at small $\rho$, the gradient of the propagator will have no $y$-component to first order. Therefore, we can assume that $y=0$ in the propagator without significantly changing its value. This allows us to build our hemispherical shells centered on the target by shifting the coordinate system by $x^{\prime}=x-a$ and then integrating the propagator over semicircles of radius $\sqrt{\rho^{2}-x^{\prime 2}}$, holding $x^{\prime}$ constant. This eliminates the $z$-dependence, effectively

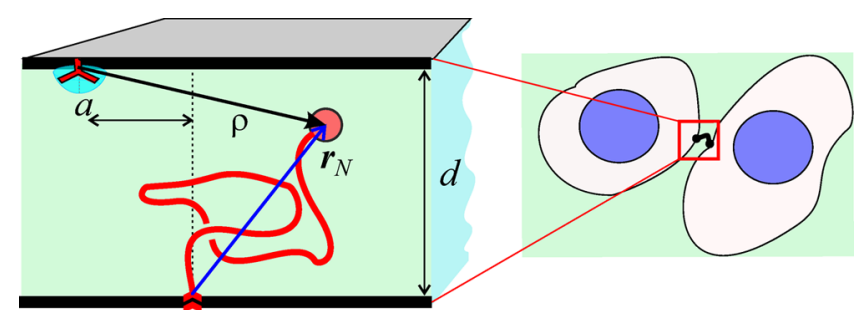

Figure 5. Schematic for the cell-adhesion process, where a flexible linker will bind to a neighboring cell across a gap $d$. The inset shows our simplified scenario: assuming the two membranes to be infinite in extent, and parallel to each other, with the receptor displaced by a distance $a$.

generating the average $\langle\sin (\pi z / d)\rangle$. The second integration is over the $x^{\prime}$-axis from $-\rho$ to $+\rho$, adding these semicircles with an appropriate surface element to recover the radial distribution function about the receptor. This distribution $P_{\text {eq }}(\rho)$ can be expanded at small $\rho$ again, exploiting the vanishing denominator as in eq 7 , and in the same way we obtain the result for the mean first passage time:

$$
\tau=\frac{2 N b^{2} d^{2}}{3 \pi^{2} D \varepsilon^{2}} \mathrm{e}^{3 a^{2} / 2 N b^{2}}
$$

One can see the same constrained dependence on the reaction volume $\varepsilon$ due to the difficulty for any chain segment to get that close to the wall. It is possible to consider the $a=0$ case using a different method, relying on the azimuthal symmetry of the propagator. We recover the nonexponential prefactor in eq 9, confirming the validity of the analysis. It is also possible to adapt this result for a very small gap, $d<\varepsilon$, where the $2 \mathrm{D}$ scaling relation $\tau \sim\left(N b^{2} / D\right) \ln \left(\sqrt{N b^{2}} / \varepsilon\right)$ replaces eq 9. See Schuss et al. ${ }^{18}$ for a freely diffusing Brownian particle analogue.

The quadratic dependence of the mean bridging time on the cell gap $d$ in eq 9 is the novel feature, but it is only valid in the tightly confined case $d \ll R_{\mathrm{g}}$. The general expression for the binding time is complicated, but the calculation in the opposite limit (short chain or wide gap: $d^{2} \gg N b^{2}$ ) is presented in Supporting Information part C. We find a very accurate interpolation formula for the mean time of bridging between two surfaces, which spans across the whole range between the two limiting cases: 

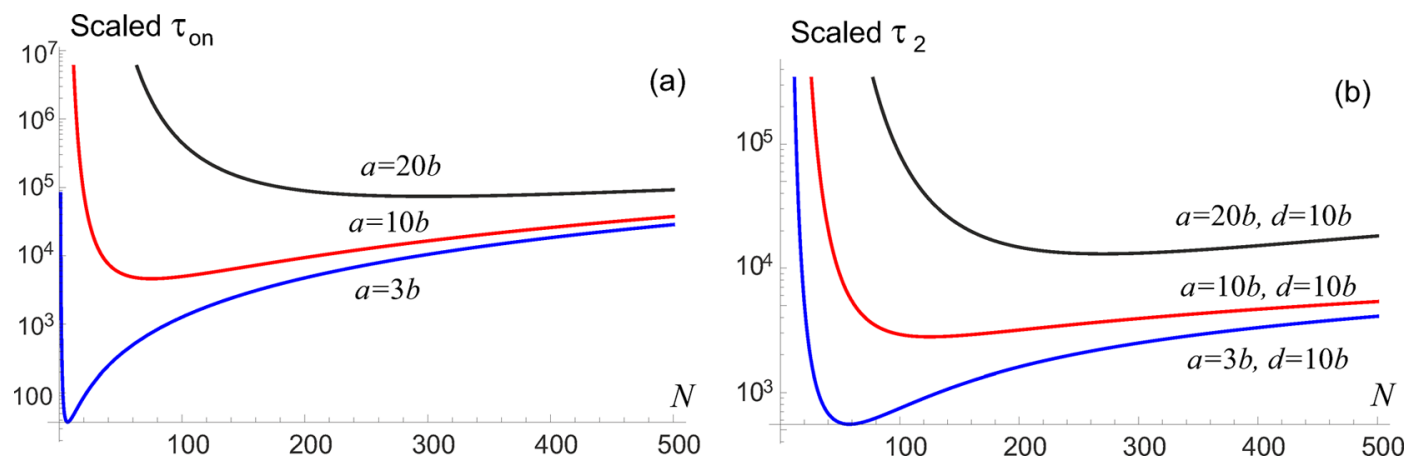

Figure 6. Dependence of scaled mean binding times $\left(\tau D \varepsilon^{-2}\right)$ on the chain length via $R_{\mathrm{g}}=\sqrt{ } \mathrm{Nb}$ : (a) for the tethered binding in the same plane, for $a$ $=3 b, 10 b, 20 b$, and (b) for bridging across the gap, for $a=3 b, 10 b, 20 b$ with $d=10 b$.

$$
\tau_{2}=\frac{2 R_{\mathrm{g}}{ }^{2} d^{2}}{3 \pi^{2} D \varepsilon^{2}} \frac{1}{\left(1+36 d^{4} / \pi^{2} R_{\mathrm{g}}{ }^{4}\right)} \mathrm{e}^{3\left(d^{2}+a^{2}\right) / 2 R_{\mathrm{g}}{ }^{2}}
$$

\section{CONCLUSIONS}

It is interesting to compare the tethered binding time $\tau_{\text {on }}$ in eq 7 with the average time for a free polymer chain to make a loop by having the last $N$ th monomer reach a sphere of radius $\varepsilon$ around the first monomer. ${ }^{9}$ The Szabo problem, corresponding to distance $a=0$ and no restricting surface-solved using our method in Supporting Information part B-is

$$
\tau_{\text {loop }}=\sqrt{\frac{\pi}{54}} \frac{\left(N b^{2}\right)^{3 / 2}}{D \varepsilon}
$$

This involves integrating the free space propagator over the full space in eq 6, rather than the constrained propagator over the half space in the single plane case. Also instructive is to compare with the average time for a free Brownian particle to escape a closed volume $V$ through a small hole of size $\varepsilon^{18}$ (the "narrow escape problem" of Holcman et al.), which is estimated as $\tau_{\text {esc }}=V / D \varepsilon$. If the volume is replaced by the average extent of chain spreading, $V=R_{\mathrm{g}}{ }^{3}$, this matches the Szabo expression in eq 11. Both have a different scaling with the size of target: $1 /$ $\varepsilon$ compared to $1 / \varepsilon^{2}$ in our eq 7 . The chain is inhibited from approaching the wall due to the polymer-specific exclusion boundary condition, and so the average time it takes to reach the target is much longer even without the additional exponential factor reflecting the entropic barrier for binding. The same argument applies to the mean bridging time $\tau_{2}$ in eq 10: an entropic repulsion of monomers from the surface causes a stronger, $1 / \varepsilon^{2}$ scaling with the receptor size.

The second factor that distinguishes the mean binding time in eqs 7 and 10 is the exponential factor: $\exp \left[3 a^{2} / 2 R_{\mathrm{g}}^{2}\right]$ and $\exp \left[3\left(a^{2}+d^{2}\right) / 2 R_{\mathrm{g}}{ }^{2}\right]$, respectively. In both cases this represents thermal activation over an entropic barrier $\Delta G=(3 / 2) k_{\mathrm{B}} T\left(a^{2} /\right.$ $N b^{2}$ ), which is essentially the free energy to stretch the chain ends by a distance $a$. This factor, significantly increasing the time for bridging to a distant target, only arises for the tethered chain. (All polymer work on the related narrow escape problems ${ }^{19,22,24}$ has thus far focused on polymers with no attachment to the boundary of the domain, which fundamentally alters the accessibility of the binding site.) One might then naively assume that the binding time will decrease monotonically as the length of the chain increases-the entropic penalty will become smaller and smaller.
However, as we find in eqs 7 and 10, there is another competing effect that decreases the rate (or increases the mean first passage time): as the chain gets longer, the effective volume that the site can explore relative to the receptor volume also increases. If we increase the chain to an infinite length, we actually return to a free particle scenario, and there is not enough confinement for the end of the chain to ever hit the receptor. Figure 6 illustrates the resulting effect, which predicts a certain optimal (shortest) binding time for any given receptor separation. It is straightforward to find the shortest binding times, and the chain length $N^{*}$ that achieves this rate in each case:

$$
\begin{aligned}
& \tau_{\text {on }}^{*} \approx 0.46 \frac{a^{4}}{D \varepsilon^{2}} \quad \text { at } N^{*}=\frac{3 a^{2}}{4 b^{2}} \\
& \tau_{2}^{*} \approx 0.04 \frac{d^{4}}{D \varepsilon^{2}} \quad \text { at } N^{*} \approx \frac{a^{2}+d^{2}}{2 b^{2}} \text { and } a \rightarrow 0
\end{aligned}
$$

The scaling with the receptor size $\varepsilon$ is an interesting feature, especially when compared with the looping or "narrow escape" problems (which both have the $\varepsilon^{-1}$ scaling). Together with the entropic barrier, this effect determines the mean first binding time of a tethered ligand. We approached this problem in a way completely different to the approach of Holcman et al. in the "narrow escape" setting. ${ }^{16,18}$ Instead of examining the Smoluchowski problem in an effective potential imposed by the constraints, we have generated the mean-field equilibrium probability density $P_{\text {eq }}(\rho)$ by integrating out the angular degrees of freedom. As a result, we were able to utilize the mean first passage time solution of Szabo et al. ${ }^{9}$ It may well be that this approach generates analytical and numerical solutions more rapidly even for the more complex problems involving potential interaction and nonideal polymer chain, as well as confined Brownian particles, since we are not having to look at the dynamical effects-only at how these affect the equilibrium effective potential.

While theoretically clean, the absorbing boundary has only limited biological application as it assumes infinitely fast binding reaction. It is more relevant to consider the case where binding to a surface is slower, and the reaction time becomes a relevant factor slowing down the reaction. For instance, DNA loop formation is much slower than the diffusive end-to-end encounter. ${ }^{33}$ In their original work on first passage time, Szabo et al. ${ }^{9}$ allowed for this by adopting a radiation condition on the flux at the boundary: $j(\varepsilon, t)=\kappa p(x, t)$. The constant $\kappa$ is indicative of the reaction rate at the boundary: for $\kappa \rightarrow \infty$ we reach the limit of an ideal absorbing boundary. In 
our formalism, accounting for $\kappa$ adds an extra term into the mean process time: $\tau_{\kappa}=\left[\kappa \varepsilon^{2} P_{\mathrm{eq}}(\varepsilon)\right]^{-1}$. For the laterally displaced receptor, the full form of the mean binding time takes the slightly modified form:

$$
\tau_{\text {on }}=\frac{N^{2} b^{4}}{9 D \varepsilon^{2}} \mathrm{e}^{3 a^{2} / 2 N b^{2}}\left(1+\frac{2 D}{\kappa \varepsilon}\right)
$$

A sufficiently small reaction volume $(\varepsilon)$ and reaction rate $(\kappa)$ could significantly slow down the binding process, in addition to the entropic penalty.

It would be interesting to apply even this approach to a practical problem of amyloid assembly, where the new peptide subunit has to bind to a specific sequence of sites by hydrogenbonding the $\beta$-sheet at the end of the existing filament, ${ }^{34}$ and the entropic barriers are explicitly reported.

\section{ASSOCIATED CONTENT}

\section{S Supporting Information}

The Supporting Information is available free of charge on the ACS Publications website at DOI: 10.1021/acs.macromol.7b01742.

Part A: calculation of radial distribution functions; part B: calculation of looping time; part C: parallel walls at large separation (PDF)

\section{AUTHOR INFORMATION}

\section{Corresponding Author}

*E-mail: emt1000@cam.ac.uk (E.M.T.).

\section{ORCID 1}

Eugene M. Terentjev: 0000-0003-3517-6578

Notes

The authors declare no competing financial interest.

\section{ACKNOWLEDGMENTS}

We appreciate helpful discussions with Preeyan Parmar and John Grenfell-Shaw. This work has been funded by EPSRC $\mathrm{EP} / \mathrm{M} 508007 / 1$

\section{REFERENCES}

(1) Vu, T.-K. H.; Hung, D. T.; Wheaton, V. I.; Coughlin, S. R. Molecular cloning of a functional thrombin receptor reveals a novel proteolytic mechanism of receptor activation. Cell 1991, 64, 10571068.

(2) Chen, J.; Ishii, M.; Wang, L.; Ishii, K.; Coughlin, S. R. Thrombin receptor activation. Confirmation of the intramolecular tethered liganding hypothesis and discovery of an alternative intermolecular liganding mode. J. Biol. Chem. 1994, 269, 16041-16045.

(3) Coughlin, S. R. Thrombin signalling and protease-activated receptors. Nature 2000, 407, 258-264.

(4) Howard, J. Mechanics of Motor Proteins and the Cytoskeleton; Sinauer Associates, Inc.: 2001.

(5) Fletcher, D. A.; Mullins, R. D. Cell mechanics and the cytoskeleton. Nature 2010, 463, 485-492.

(6) Jeppesen, C.; Wong, J. Y.; Kuhl, T. L.; Israelachvili, J. N.; Mullah, N.; Zalipsky, S.; Marques, C. M. Impact of polymer tether length on multiple ligand-receptor bond formation. Science 2001, 293, 465-468.

(7) Wong, J. Y.; Kuhl, T. L.; Israelachvili, J. N.; Mullah, N.; Zalipsky, S. Direct measurement of a tethered ligand-receptor interaction potential. Science 1997, 275, 820-822.

(8) Wilemski, G.; Fixman, M. Diffusion-controlled intrachain reactions of polymers. I theory. J. Chem. Phys. 1974, 60, 866-877.

(9) Szabo, A.; Schulten, K.; Schulten, Z. First passage time approach to diffusion controlled reactions. J. Chem. Phys. 1980, 72, 4350-4357.
(10) Pastor, R. W.; Zwanzig, R; Szabo, A. Diffusion limited first contact of the ends of a polymer: comparison of theory with simulation. J. Chem. Phys. 1996, 105, 3878-3882.

(11) Göndör, A.; Ohlsson, R. Chromosome crosstalk in three dimensions. Nature 2009, 461, 212-217.

(12) Deng, W.; Lee, J.; Wang, H.; Miller, J.; Reik, A.; Gregory, P. D.; Dean, A.; Blobel, G. A. Controlling long-range genomic interactions at a native locus by targeted tethering of a looping factor. Cell 2012, 149, $1233-1244$.

(13) Vafabakhsh, R.; Ha, T. Extreme bendability of DNA less than 100 base pairs long revealed by single-molecule cyclization. Science 2012, 337, 1097-1101.

(14) Waters, J. T.; Kim, H. D. Equilibrium Statistics of a SurfacePinned Semiflexible Polymer. Macromolecules 2013, 46, 6659-6666.

(15) Bressloff, P. C.; Newby, J. M. Stochastic models of intracellular transport. Rev. Mod. Phys. 2013, 85, 135-196.

(16) Holcman, D.; Schuss, Z. The narrow escape problem. SIAM Rev. 2014, 56, 213-257.

(17) Singer, A.; Schuss, Z.; Holcman, D.; Eisenberg, R. S. Narrow escape, part I. J. Stat. Phys. 2006, 122, 437-463.

(18) Schuss, Z.; Singer, A.; Holcman, D. The narrow escape problem for diffusion in cellular microdomains. Proc. Natl. Acad. Sci. U. S. A. 2007, 104, 16098-16103.

(19) Muthukumar, M.; Baumgärtner, A. Effects of entropic barriers on polymer dynamics. Macromolecules 1989, 22, 1937-1941.

(20) Schweizer, K. S.; Saltzman, E. J. Entropic barriers, activated hopping, and the glass transition in colloidal suspensions. J. Chem. Phys. 2003, 119, 1181-1196.

(21) Sung, W.; Park, P. J. Polymer translocation through a pore in a membrane. Phys. Rev. Lett. 1996, 77, 783-786.

(22) Muthukumar, M. Polymer translocation through a hole. J. Chem. Phys. 1999, 111, 10371-10374.

(23) Knowles, T. P. J.; Shu, W.; Devlin, G. L.; Meehan, S.; Auer, S.; Dobson, C. M.; Welland, M. E. Kinetics and thermodynamics of amyloid formation from direct measurements of fluctuations in fibril mass. Proc. Natl. Acad. Sci. U. S. A. 2007, 104, 10016-10021.

(24) Bicout, D. J.; Szabo, A. Entropic barriers, transition states, funnels, and exponential protein folding kinetics: a simple model. Protein Sci. 2000, 9, 452-465.

(25) Doi, M.; Edwards, S. F. The Theory of Polymer Dynamics; Oxford University Press: 1988.

(26) DiMarzio, E. A. Proper accounting of conformations of a polymer near a surface. J. Chem. Phys. 1965, 42, 2101-2106.

(27) Edwards, S. F.; Freed, K. F. The entropy of a confined polymer. J. Phys. A: Gen. Phys. 1969, 2, 145-150.

(28) Joanny, J.-F.; Leibler, L.; De Gennes, P. G. Effects of polymer solutions on colloid stability. J. Polym. Sci., Polym. Phys. Ed. 1979, 17, 1073-1084.

(29) de Gennes, P.-G. Conformations of polymers attached to an interface. Macromolecules 1980, 13, 1069-1075.

(30) Bickel, T.; Jeppesen, C.; Marques, C. M. Local entropic effects of polymers grafted to soft interfaces. Eur. Phys. J. E: Soft Matter Biol. Phys. 2001, 4, 33-43.

(31) Kramers, H. A. Brownian motion in a field of force and the diffusion model of chemical reactions. Physica 1940, 7, 284-304.

(32) Chandrasekhar, S. Stochastic problems in physics and astronomy. Rev. Mod. Phys. 1943, 15, 1-89.

(33) Vologodskii, A.; Frank-Kamenetskii, M. D. Strong Bending of the DNA Double Helix. Nucleic Acids Res. 2013, 41, 6785-6792.

(34) Buell, A. K.; Dhulesia, A.; White, D. A.; Knowles, T. P.; Dobson, C. M.; Welland, M. E. Detailed analysis of the energy barriers for amyloid fibril growth. Angew. Chem., Int. Ed. 2012, 51, 5247-5251. 